\title{
DISTRIBUTIONS DES SINISTRES INCENDIE SELON LEUR COUT
}

\author{
Giovanna Ferrara \\ Italie
}

\section{INTRODUCTION}

On peut penser que la dimension des sinistres est la résultante d'un très grand nombre de causes indépendantes à effets positifs. Soient

$$
\xi_{1}, \xi_{2}, \ldots, \xi_{n}
$$

les variables aléatoires indépendantes qui représentent les facteurs élementaires exerçant leur action dans l'ordre indiqué par les indices.

Si $X_{v}$ est l'ampleur du sinistre dû aux facteurs $\xi_{1}, \xi_{2}, \ldots, \xi_{v}$ nous pouvons supposer que l'augmentation causée par $\xi_{v+1}$ soit proportionelle à $\xi_{v+1}$ et à une certaine fonction, $g\left(X_{v}\right)$ de la dimension $X_{\nu}$.

C'est à dire, nous supposons qu'il y ait les relations:

$$
X_{v+1}=X_{v}+\xi_{v}+1 g\left(X_{v}\right)
$$

où

$$
\begin{aligned}
& \nu=0, \mathrm{I}, \ldots, n-\mathrm{I} \\
& X_{0}=0 ; \quad g\left(X_{0}\right)=\mathrm{I}
\end{aligned}
$$

Il s'ensuit que

$$
\xi_{1}+\xi_{2}+\ldots+\xi_{n}=\sum_{0}^{n-1} \frac{X_{v+1}-X_{\nu}}{g\left(X_{\nu}\right)}
$$

Si chaque facteur n'apporte qu'un faible contribut à l'augmentation du sinistre, nous pouvons alors poser approximativement aussi :

$$
\xi_{1}+\xi_{2}+\ldots+\xi_{n}=\int_{x_{0}}^{x} \frac{d t}{g(t)}
$$


Comme $X$ varie de $X_{0}$ à $X_{n}$ (et que $X=X_{n}$ désigne la dimension extrème du sinistre) le second membre de la (2) décrit une variable aléatoire, fonction de $X$.

Puisque par hypothèse $\xi_{1}, \xi_{2}, \ldots, \xi_{n}$ sont des variables aléatoires indépendante, si $n$ est suffisament grand, d'après le théorème de la limite des probabilités il s'insuit que la variable aléatoire au second membre de la (2), est, à la limite, distribuée suivant la loi normale. Si l'on pose $g(t)=t-c$, la variable aléatoire normale qui décrit la dimension du sinistre pourra être posée dans la forme $\ln (X-c)$.

Si $Z^{*}$ est une variable aléatoire normale réduite $(0, \mathrm{I})$, entre $Z^{*}$ et $X$ il y aura la relation

$$
Z^{*}=\frac{\ln (X-c)-m}{\sigma}
$$

C'est à dire en posant $\alpha=\mathrm{I} / \sigma ; \beta=-m / \sigma$

$$
Z^{*}=\alpha \ln (X-c)+\beta
$$

d'où

$$
X=c+\exp \frac{Z^{*}-\beta}{\alpha}
$$

La fonction cumulative de la variable aléatoire $X$ sera pour $x>c$

$$
\begin{aligned}
F(x) & =\operatorname{Prob}\{X \leqslant x\}=\operatorname{Prob}\left\{c+\exp Z^{*}-\beta \leqslant x\right\}= \\
& =\operatorname{Prob}\left\{Z^{*} \leqslant \alpha \ln (x-c)+\beta\right\}
\end{aligned}
$$

c'est à dire

$$
F(x)=\frac{\mathrm{I}}{\sqrt{2 \pi}} \int_{-\infty}^{\alpha \ln (x-c)+\beta} \exp \left\{-\frac{t^{2}}{2}\right\} d t
$$

et la fonction de densité

$$
f(x)=\frac{\mathrm{I}}{\sqrt{2 \pi}} \frac{\alpha}{x-c} \exp -\frac{\mathrm{I}}{2}\{\alpha \ln (x-c)+\beta\}^{2}
$$


Pour les développements successifs il est préférable de considérer la variable normale $Z\left(0 ; \frac{\mathrm{r}}{\sqrt{2}}\right)$ liée à $Z *$ par la relation

$$
Z^{*}=\frac{Z}{\sqrt{2}}
$$

c'est à dire

où

$$
\begin{aligned}
& Z=a \ln (x-c)+b \\
& a=\frac{\alpha}{\sqrt{2}} ; \quad b=\frac{\beta}{\sqrt{2}}
\end{aligned}
$$

La fonction de densité de $Z$ est:

$$
f(z)=\frac{\mathrm{I}}{\sqrt{\pi}} \exp \left(-z^{2}\right)
$$

et celle de $X$

$$
f(x)=\frac{\mathrm{I}}{V / \pi} \cdot \frac{a}{x-c} \exp -\{a \ln (x-c)+b\}^{2} \quad(8 \text { bis })
$$

Les trois premiers moments par rapport à o de la variable aléatoire $X$ en fonction de $Z$ seront:

$$
\begin{aligned}
\mu_{1} & =c+\frac{\mathrm{I}}{\sqrt{\pi}} \int_{-\infty}^{+\infty} \exp \left(\frac{z-b}{a}\right) \exp \left(-z^{2}\right) d z \\
& =c+\exp \left(\frac{\left.\mathrm{I}-\frac{4 a b}{4 a^{2}}\right)}{\mu_{2}}=c^{2}+\left(\mu_{1}-c\right)^{2} \exp \left(\frac{\mathrm{I}}{2 a^{2}}\right)+2 c\left(\mu_{1}-c\right)\right. \\
\mu_{3} & =c^{3}+\left(\mu_{1}-c\right)^{3} \exp \left(\frac{3}{2 a^{2}}\right)+3 c^{2}\left(\mu_{1}-c\right)+3 c\left(\mu_{1}-c\right)^{2} \exp \left(\frac{\mathrm{I}}{2 a^{2}}\right)
\end{aligned}
$$

Pour l'écart absolu moyen par rapport à la moyenne, on aura:

$$
S=\int_{-\infty}^{+\infty}\left|x-\mu_{1}\right| f(x) d x=2 \int_{-\infty}^{\mu_{1}}\left(\mu_{1}-x\right) f(x) d x
$$


puisque pour $x=\mu_{1} ; \quad z=\mathrm{I} / 4 a$

$$
S=\frac{2}{\sqrt{\pi}} \int_{-\infty}^{+1 / 4 a} \exp \frac{\mathrm{I}-4 a b}{4 a^{2}}-\exp \frac{z-b}{a} \cdot d z
$$

d'où vient:

$$
S=\frac{2\left(\mu_{1}-c\right)}{\sqrt{\pi}} \int_{-1 / 4 a}^{+1 / 4 a} \exp \left(-t^{2}\right) d t
$$

rappelant la fonction connue:

$$
\Theta(\lambda)=\frac{\mathrm{I}}{\sqrt{\pi}} \int_{-\lambda}^{+\lambda} \exp \left(-t^{2}\right) d t
$$

la relation précédente devient:

$$
S=2\left(\mu_{1}-c\right) \Theta\left(\frac{I}{4 a}\right)
$$

Le coefficient de variation suivant l'écart absolu moyen est donc:

$$
\frac{S}{\mu_{1}}=\frac{2\left(\mu_{1}-c\right)}{\mu_{1}} \Theta\left(\frac{\mathrm{I}}{4 a}\right)
$$

2. Ajustement des données italiennes INCENdie À LA LOI LoGNORMALE

D'après recherches faites sur les données du ,Concordato Italiano Incendi Rischi Industriali" pour la période $1963-1965$, il est possible de tirer la distribution des sinistres selon leur coût soit pour l'ensemble des risques assurés soit pour les principaux regroupements des industries effectués suivant l'activité excrcée et suivant le capital assuré.

Ces distributions présentent quelques particularités permettant de supposer que l'ampleur des sinistres suit la loi log-normale.

Il s'agit en fait de distributions unimodales, dissymétriques, étalées vers la droite: le $50 \%$ des sinistres est en général concentré dans les deux premières classes; tandis qu'ils diminuent progressivement dans les autres.

La fonction d'ajustement est donc donnée par la ( 8 bis). 
Pour déterminer la valeur des paramètres, on a consideré la variable $Z$ liée à $X$ par la relation ( 4 bis).

Puisque $Z$ est une variable aléatoire normale, les fréquences cumulées, c'est à dire les fréquences des valeurs de $Z$ inférieures à $z_{i}$, sont exprimées par

$$
\frac{\mathbf{I}}{\sqrt{\pi}} \int_{-\infty}^{z_{i}} \exp \left(-z^{2}\right) d z
$$

Désignant par $P\left(x_{i}\right)$ les fréquences cumulées de $X$ (c'est à dire les fréquences des valeurs de $X$ inférieure a $x_{i}$ ) on peut poser

$$
P\left(x_{i}\right)=\frac{\mathrm{I}}{\sqrt{\pi}} \int_{-\infty}^{z_{i}} \exp \left(-z^{2}\right) d z
$$

Après avoir établi les valeurs $z_{i}$, on a considéré les relations

$$
z_{i}=a \ln \left(x_{i}-c\right)+b
$$

Le paramètre $c$ qui se présente en forme non linéaire a été déterminé par tâtonnements, en prenant comme valeur de départ la quantité $c$ d'après l'égalité:

$$
\frac{z_{3}-z_{2}}{z_{2}-z_{1}}=\frac{\ln \left(x_{3}-c\right)-\ln \left(x_{2}-c\right)}{\ln \left(x_{2}-c\right)-\ln \left(x_{1}-c\right)}
$$

concernant les trois premières classes de sinistres.

Les paramètres $a$ et $b$ ont été déterminés par la méthode des moindres carrés.

D'après les valeurs établies suivant la (I7), par l'emploi de la (I6) on a trouvé les valeurs théoriques $P^{*}\left(x_{i}\right)$ et $f^{*}\left(x_{i}\right)$.

Pour apprécier l'ajustement on a calculé l'écart moyen entre les valeurs théoriques et les valeurs observées, c'est à dire

et l'indice $\chi^{2}$.

$$
I=\sum_{i}^{n}\left|f\left(x_{i}\right)-f^{*}\left(x_{i}\right)\right|
$$

De plus la moyenne et le coefficient de variation selon l'écart absolu moyen des distributions observées ont été comparés à ceux de l'application des relations (9) et ( 15 ).

Nous reportons en appendice les phases du déroulement d'ajustement concernant la categorie des industries métallurgiques. 


\section{Analyse des résultats obtenus}

A) Catégories d'industries selon leur activité

L’ajustement à la loi log-normale des distributions des sinistres incendie suivant leur coût peut être considéré en général satisfaisant.

Les valeurs de $\chi^{2}$ sont pour toutes les catégories inférieures à la valeur théorique tabulée au seuil de probabilité de Io \%.

L'écart moyen entre les valeurs observées et les valeurs ajustées est uniquement élevé pour les industries des combustibles (23\%). De plus dans cette distribution la moyenne théorique s'éloigne dans une certaine mesure de la moyenne observée et il en est de même pour le coefficient de variation selon l'écart absolu moyen.

Il faut noter cependant que les sinistres de cette catégorie ne sont qu'une modeste partie de l'ensemble des sinistres (seulement le I, $3 \%$ ) et c'est pourquoi la moyenne relevée a un degré de précision très bas.

En ce qui concerne les industries de l'alimentation et du cuir les ajustements peuvent être acceptables bien que les valeurs effectives de la moyenne et du coefficient de variation s'éloignent des valeurs théoriques.

\section{B) Catégories d'industries selon le capital assuré}

Si l'on regroupe les données des sinistres suivant le capital assuré, les distributions des sinistres selon leur coût présentent un ajustement moins satisfaisant à la loi log-normale.

Les valeurs de $\chi^{2}$ sont élevées excepté la classe des risques avec un capital supérieur à I.00o millions.

L'écart moyen entre les valeurs théoriques et les valeurs observées est cependant relativament bas; les moyennes et les coefficients de variation sont suffisamment proches. 


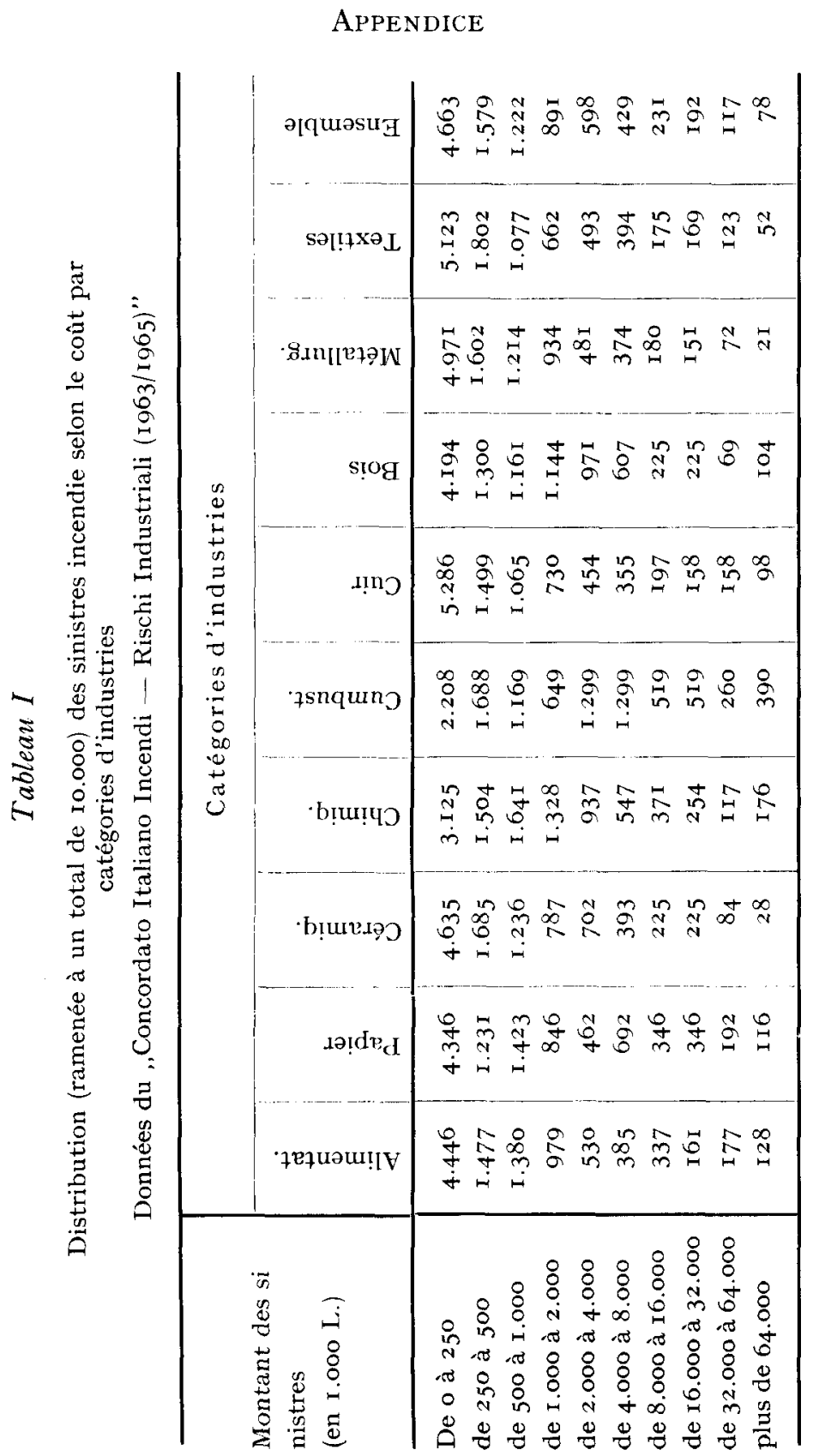









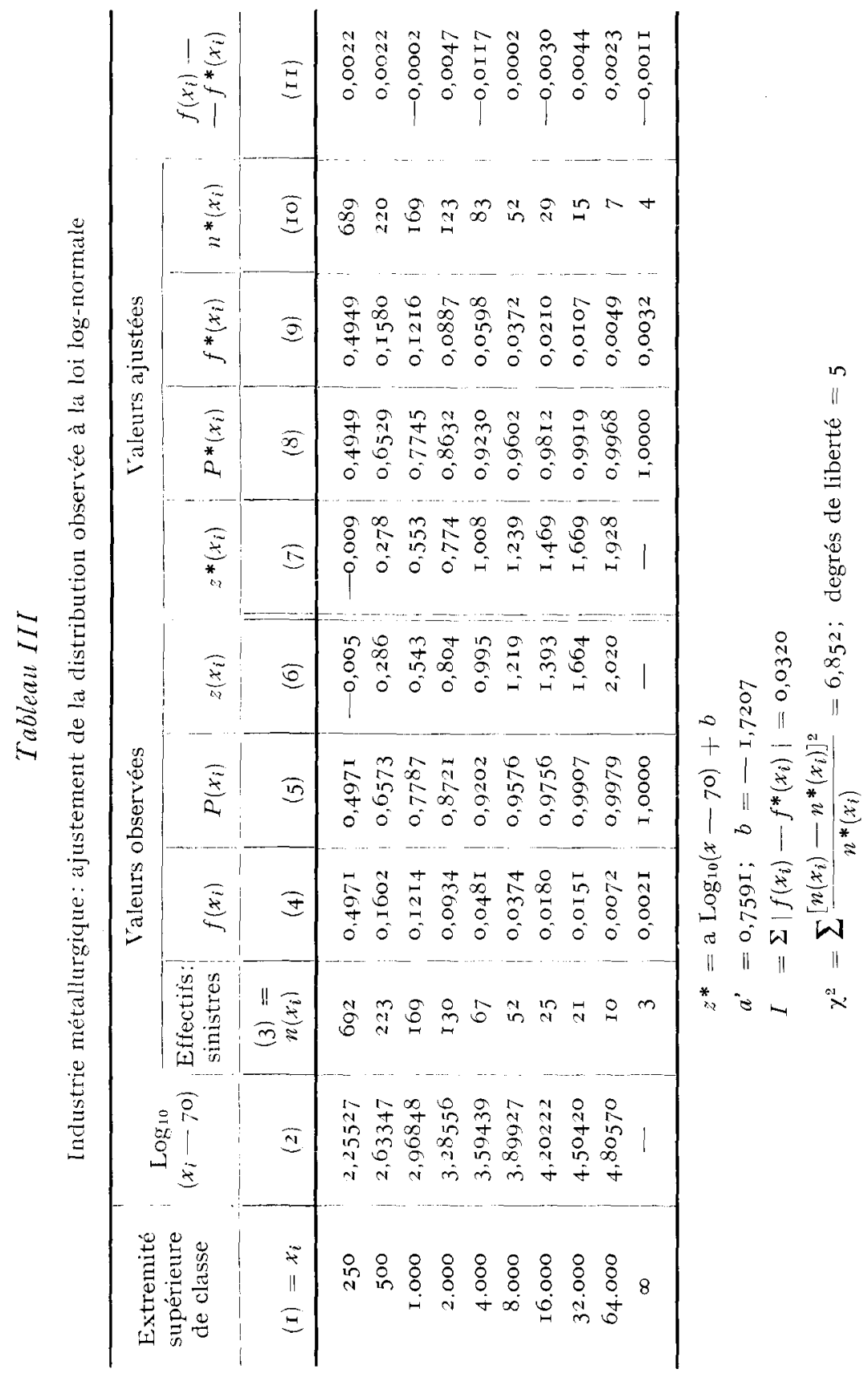




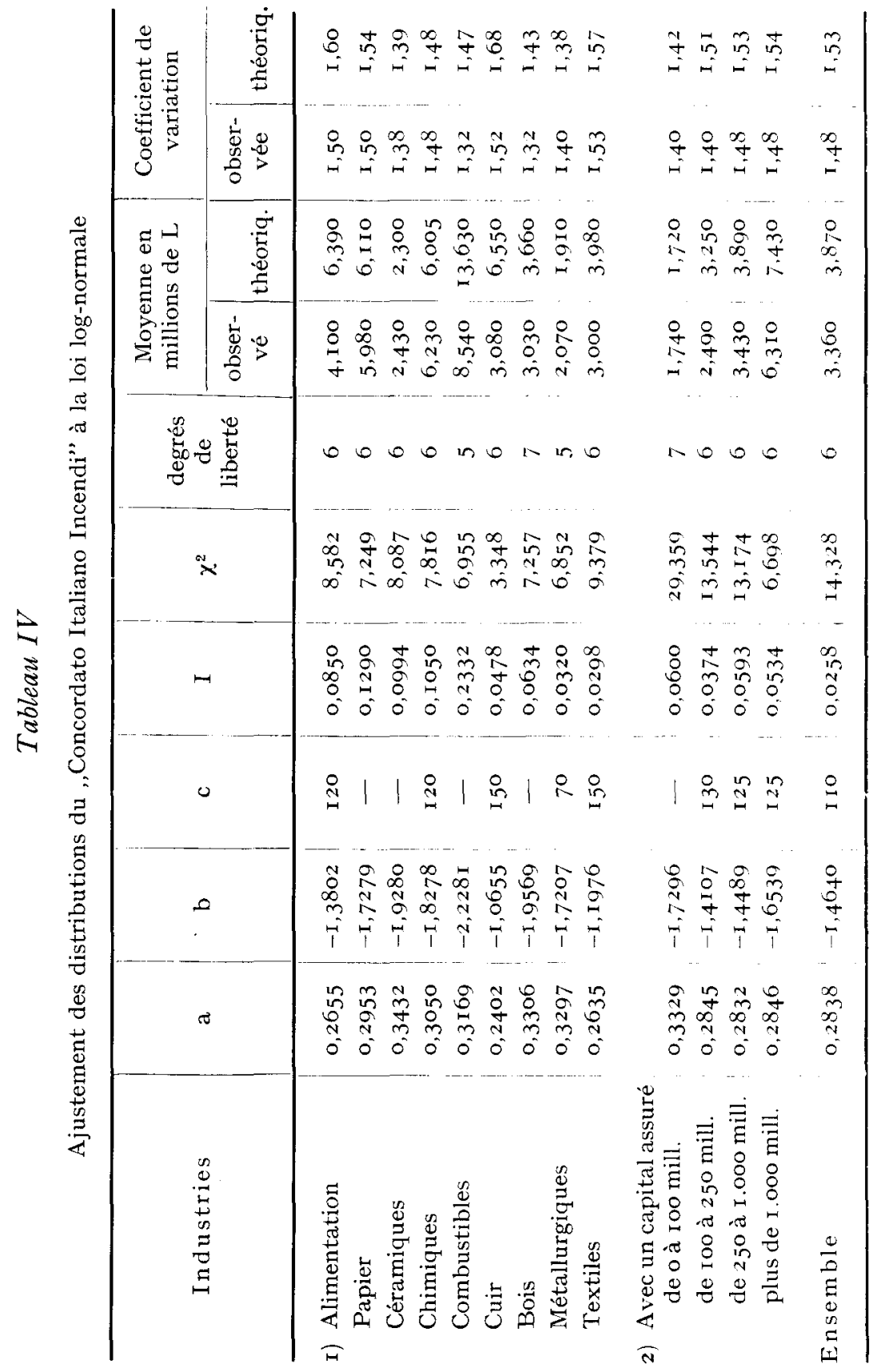



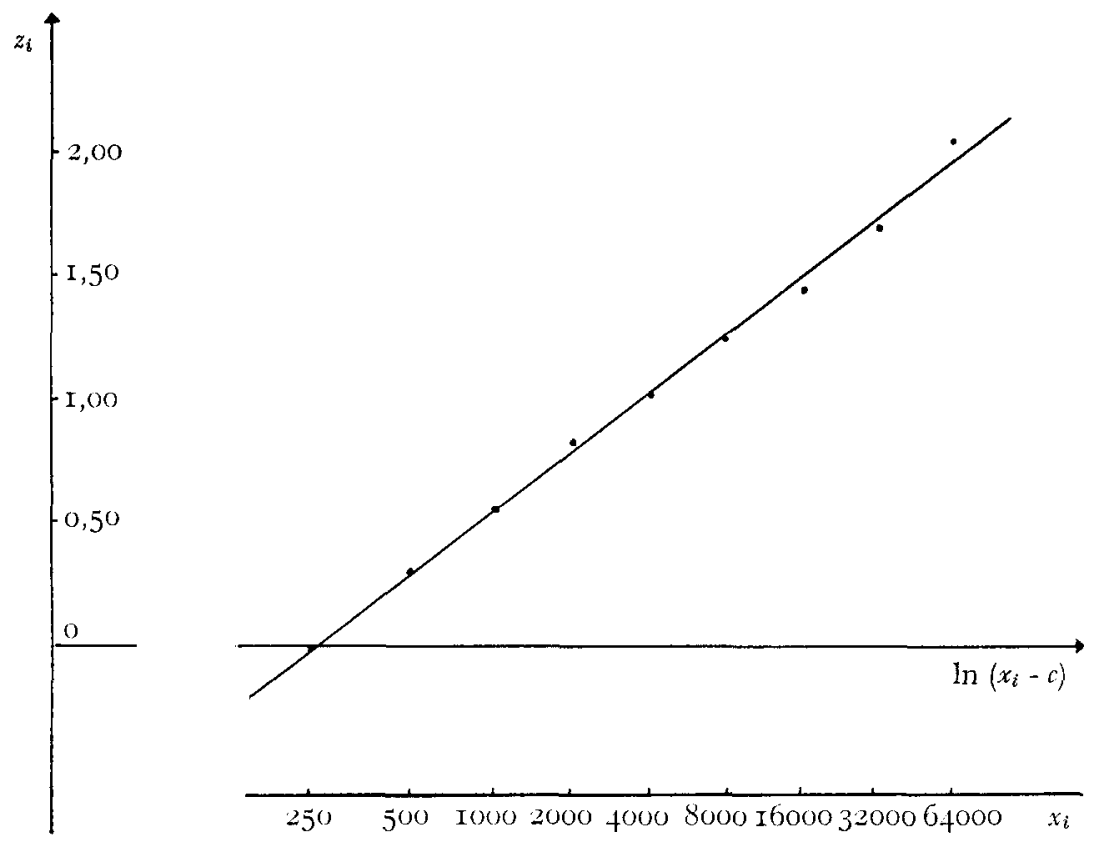

INDLSTRIE METALlurgigue: Ajustement de la distribution des sinistres incendie selon le coût à la loi log-normale. Représentation grafique de la variable $Z . Z=a \ln (X-c)+b ; a=0,3297 b=-\mathbf{1}, 7107 ; c=70$

Source: „Concordato Italiano Incendi - Rischi Industriali” Données de la période I963-I965. Elaboration du Service Etudes des Assicurazioni Generali 